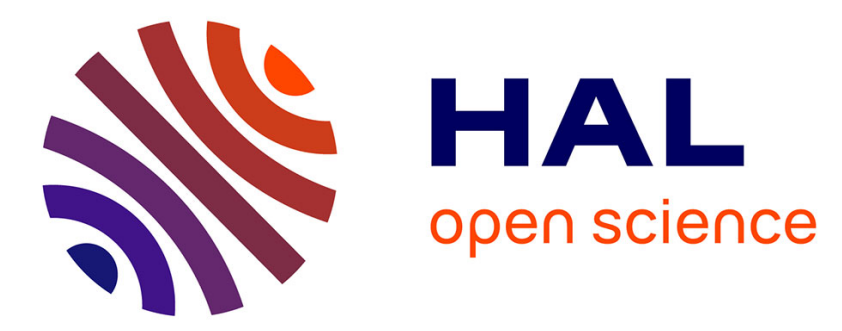

\title{
Transitions à plusieurs quanta, étudiées dans le référentiel tournant
}

\author{
Ennio Arimondo, M. Cassettari, G. Salvetti
}

\section{To cite this version:}

Ennio Arimondo, M. Cassettari, G. Salvetti. Transitions à plusieurs quanta, étudiées dans le référentiel tournant. Journal de Physique, 1971, 32 (7), pp.573-578. 10.1051/jphys:01971003207057300 . jpa00207112

\section{HAL Id: jpa-00207112 https://hal.science/jpa-00207112}

Submitted on 1 Jan 1971

HAL is a multi-disciplinary open access archive for the deposit and dissemination of scientific research documents, whether they are published or not. The documents may come from teaching and research institutions in France or abroad, or from public or private research centers.
L'archive ouverte pluridisciplinaire HAL, est destinée au dépôt et à la diffusion de documents scientifiques de niveau recherche, publiés ou non, émanant des établissements d'enseignement et de recherche français ou étrangers, des laboratoires publics ou privés. 
Classification

Physics Abstracts :

18.52

\title{
TRANSITIONS A PLUSIEURS QUANTA, ÉTUDIÉES DANS LE RÉFÉRENTIEL TOURNANT (*)
}

\author{
E. ARIMONDO
}

Istituto di Fisica dell'Università, Pisa

M. CASSETTARI et G. SALVETTI

Laboratorio di Fisica Atomica e Molecolare del C. N. R., Pisa

(Reçu le 20 janvier 1971, révisé le 9 mars 1971)

\begin{abstract}
Résumé. - L'intensité et le déplacement des transitions à plusieurs quanta avec photons $\sigma$ et $\pi$, sont examinés dans le DPPH, en détectant le moment cinétique absorbé par l'échantillon. Ces quantités sont étudiées en fonction de l'intensité du champ oscillant parallèlement au champ statique. On compare les résultats expérimentaux aux courbes théoriques obtenues en résolvant les équations de Bloch pour le système de spin dans le référentiel tournant. En considérant l'effet de superposition entre les différentes raies de résonance, on obtient un accord excellent entre la théorie et l'expérience.
\end{abstract}

Abstract. - The intensity and shift of many-quantum transitions involving $\sigma$ and $\pi$ photons have been examined in the DPPH by the angular momentum detection, as a function of the intensity of the applied $\pi$ radiofrequency field. The resonances are interpreted by solving the Bloch equations in the rotating frame. Keeping into account the superposition effect between the resonance lines, a very good agreement is found between the theory and the experimental results.

Introduction. - Dans une expérience de résonance magnétique un système de spins de rapport gyromagnétique $\gamma$ est soumis à un champ statique $H_{0}$ et à un champ radiofréquence $H_{1}$, perpendiculaire à $H_{0}$ et tournant à vitesse angulaire $\omega$. Il y a absorption de puissance et de moment cinétique par les spins lorsque la condition de résonance $H_{0}=-(\omega / \gamma)$ est satisfaite. Dans un référentiel solidaire avec $H_{1}$, les spins sont soumis à un champ statique effectif

$$
H_{\mathrm{c}}=\left[\left(H_{0}+\frac{\omega}{\gamma}\right)^{2}+H_{1}^{2}\right]^{1 / 2} \text {. }
$$

Si l'on applique un champ oscillant $\mathcal{J}_{1} \cos \Omega t$ parallèlement au champ statique des nouvelles résonances vont apparaître lorsque la condition $H_{\mathrm{e}}=-(n \Omega / \gamma)$ est satisfaite ( $n$ entier quelconque).

Ces résonances peuvent être interprétées comme résonances à plusieurs quanta par les spins quantifiés dans le référentiel tournant, dues aux composantes perpendiculaire et parallèle à $H_{\mathrm{e}}$ du champ $\mathfrak{H}_{1}$, ces composantes représentant respectivement dans le référentiel tournant les photons $\sigma$ et $\pi$.

Ce point de vue est équivalent à considérer les transitions à plusieurs quanta $\omega+n \Omega$ dans le réfé-

(*) Ce travail a été supporté par Gruppo Nazionale Struttura della Materia du C. N. R., Pisa. rentiel du laboratoire ; néanmoins on utilise la description dans le référentiel tournant puisque elle donne une vision directe des aspects physiques de ces résonances [1].

Bien qu'une grande attention ait été réservée à l'observation des résonances, il n'en est pas de même pour leur déplacement radiatif qui a été examiné seulement par Lhote, Motchane et Théobald sur un charbon de dextrose [2]. L'expression du déplacement radiatif en fonction de l'intensité $\mathcal{H}_{1}$ du champ oscillant peut être déterminée dans une manière très simple, reportée par Abragam [3]. Dans le référentiel tournant la composante du champ $\mathfrak{H}_{1}$ perpendiculaire au champ effectif, peut être décomposée en deux champs tournants; l'un donne lieu à la résonance magnétique, l'autre à un déplacement Bloch-Siegert. Si $\mathfrak{H}_{1} \ll H_{\mathrm{e}}$ le déplacement de la fréquence de résonance est :

$$
\Delta \Omega=\frac{\gamma \mathcal{H}_{1}^{2}}{16} \cdot \frac{H_{1}^{2}}{\left[\left(H_{0}+\frac{\omega}{\gamma}\right)^{2}+H_{1}^{2}\right]^{3 / 2}} .
$$

Nous nous proposons dans ce travail d'étudier le déplacement radiatif de ces résonances à plusieurs quanta en détectant le moment cinétique absorbé par l'échantillon [4]. Par cette méthode de détection on examine le couple agissant sur l'échantillon dans la direction $\mathrm{Oz}$ du champ statique, couple qui est égal à 
la valeur moyenne sur le temps du produit vectoriel entre l'aimantation $M$ de l'échantillon et le champ magnétique $\underline{H}$ appliqué :

$$
C=\overline{(M \wedge \underline{H})_{z}} \text {. }
$$

Dans les expériences de résonance magnétique aux bas champs statiques et lorsque des champs radiofréquence très intenses sont appliqués à l'échantillon, cette méthode se révèle avantageuse comparée aux méthodes usuelles de détection. On a alors observé la "résonance rotatoire " avec des champs radio-fréquence dont l'intensité est du même ordre de l'intensité du champ statique $H_{0}$. Dans ces conditions l'expression (1) n'est plus valable et il a été nécessaire d'obtenir une expression théorique exacte pour le déplacement de la résonance sous l'effet du champ radiofréquence appliqué.

Le phénomène de la «résonance rotatoire " a été considéré la première fois par Redfield [5] en utilisant l'hypothèse de la température de spin pour décrire la conduite des spins dans le référentiel tournant. Dans le cas où les équations de Bloch sont valables, elles ont été utilisées pour interpréter les résultats des expériences de résonance magnétique rotatoire soit nucléaire que électronique. Diverses méthodes ont été employées pour résoudre ces équations, à savoir un développement en série [6] et une solution numérique [7].

Les expériences ont été faites sur le radical libre 2,2 diphényl-1-picryl-hydrasyl (DPPH) pour lequel les équations de Bloch sont valables. On va donner une solution de ces équations par une méthode originale de solution pour interpréter les résultats de nos expériences à très grands champs de radiofréquence.

II. Etude théorique. - Dans les cas où le champ $H_{1}$ est d'intensité élevée et $T_{2} \ll T_{1}$, l'hypothèse de la température de spin dans le référentiel tournant est généralement utilisée pour interpéter les résultats des expériences de résonance magnétique effectuées dans les solides. On suppose que dans ce référentiel les spins atteignent d'abord un équilibre thermique dans le champ $H_{\mathrm{e}}$ appliqué, et qu'ils relaxent ensuite sous l'action du réseau. Pour le DPPH $T_{1}=T_{2}=T$ et on ne peut pas utiliser cette hypothèse puisque il y a en même temps relaxation entre les spins et le réseau. Dans ce cas la résolution des équations de Bloch donne une description complète des phénomènes, en particulier, pour ce qui nous concerne, de l'absorption par les spins quantifiés dans le champ $H_{\mathrm{e}}$, en appliquant le champ oscillant parallèlement de $H_{0}$.
La méthode expérimentale utilisée permet d'examiner la résonance lorsque les intensités des champs de radiofréquence sont du même ordre que $H_{\mathrm{c}}$. Dans ces conditions il est nécessaire de résoudre les équations de Bloch modifiées, où l'on suppose que l'aimantation relaxe dans le champ magnétique instantané $\underline{H}_{0}+\underline{H}(t)$.

Considérons un spin électronique $1 / 2$ soumis au champ statique $H_{0}$ dans la direction de l'axe $\mathrm{Oz}$ et à deux champs magnétiques de radiofréquence, le premier d'amplitude $H_{1}$ tournant avec une pulsation $\omega$ dans le plan xy, l'autre $\mathfrak{H}_{1} \cos \Omega t$ oscillant parallèlement à $\mathrm{Oz}$. Dans le référentiel tournant à la vitesse angulaire $\omega$ autour de $\mathrm{Oz}$ les équations de Bloch sont

$$
\begin{aligned}
& \frac{\mathrm{d} M}{\overline{\mathrm{d} t}}=-\underline{\gamma M} \wedge\left(\underline{H}_{\mathrm{e}}+\underline{H}_{1} \cos \Omega t\right)-
\end{aligned}
$$

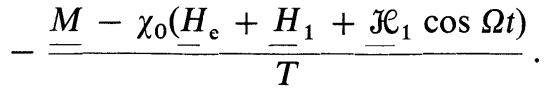

La solution de ces équations peut être cherchée en décomposant l'aimantation en ses composantes transversale $m_{+}=M_{x}+i M_{y}$ et longitudinale $M_{z}$ :

$m_{+}=A \exp -\left[\frac{1}{T}(1+i \Delta \omega T) t-\imath \frac{\Omega_{1}}{\Omega} \sin \Omega t\right] /$

$M_{z}=\sum_{K} M_{z K} \mathrm{e}^{i K \Omega t}$

où l'on a posé $\omega_{0}=-\gamma H_{0}, \omega_{1}=-\gamma H_{1}$, $\Omega_{1}=-\gamma \mathcal{H}_{1}$ et $\Delta \omega=\omega-\omega_{0}$.

Les équations (2) sont usuellement résolues en retenant seulement les premiers termes dans le développement de $M_{z}$, ce qui équivaut à considérer la solution aux ordres les plus bas en $\omega_{1} / \Omega$ et $\Omega_{1} / \Omega$ [6], [9]. Si on cherche une expression complète pour le déplacement radiatif, cette approximation n'est pas suffisante et l'on doit utiliser toutes les harmoniques dans l'expression de l'aimantation $M_{z}$. On obtient alors un système avec un nombre infini d'équations, une pour chacune des composantes harmoniques.

Pour simplifier l'exposé, nous allons résoudre ce système dans le cas plus simple des équations de Bloch non modifiées, lorsque $\underline{M}$ relaxe seulement vers $\chi_{0} \underline{H}_{0}$. La solution générale des équations modifiées (2) est plus laborieuse, mais ne présente pas des difficultés supplémentaires et l'expression du couple que nous écrirons est déduite de cette solution-là.

En utilisant pour $m_{+}$l'expression (3), on obtient les parties réelle, $\operatorname{Re}\left(M_{z K}\right)$ et imaginaire, $\operatorname{Im}\left(M_{z K}\right)$, de la composante à la fréquence $K \Omega$ de l'aimantation longitudinale :

$$
\begin{gathered}
\operatorname{Re}\left(M_{z K}\right)=-\frac{\omega_{1}^{2} T^{2}}{1+K^{2} \Omega^{2} T^{2}} \sum_{n} \frac{1}{2 D_{n}}\left\{\left[\alpha_{n K}+\beta_{n K} K \Omega T(\Delta \omega+n \Omega) T\right] E_{n}+\right. \\
\left.\quad+\left[\alpha_{n K}(\Delta \omega+n \Omega) T+\beta_{n K} K \Omega T\right] F_{n}\right\} \\
\begin{array}{c}
\operatorname{Im}\left(M_{z K}\right)=\frac{\omega_{1}^{2} T^{2}}{1+K^{2} \Omega^{2} T^{2}} \sum_{n} \frac{1}{2 D_{n}}\left\{\left[\alpha_{n K} K \Omega T-\beta_{n K}(\Delta \omega+n \Omega) T\right] E_{n}+\right. \\
\left.+\left[\alpha_{n K} K \Omega T(\Delta \omega+n \Omega) T+\beta_{n K}\right] F_{n}\right\} .
\end{array}
\end{gathered}
$$


On a posé :

$$
\alpha_{n K}=J_{-(K+n)}(x)+J_{K-n}(x) ;
$$$$
\beta_{n K}=J_{-(K+n)}(x)-J_{K-n}(x)
$$

où $J_{n}(x)$ est la fonction de Bessel du premier type d'ordre $n$ de l'argument $x=\Omega_{1} / \Omega$. On a encore posé

$$
\begin{aligned}
& E_{n}=\sum_{K} J_{K-n}(x) \operatorname{Re}\left(M_{z k}\right) . \\
& F_{n}=\sum_{K} J_{K-n}(x) \operatorname{Im}\left(M_{z k}\right)
\end{aligned}
$$

et

$$
D_{n}=1+(\Delta \omega+n \Omega)^{2} \mathrm{~T}^{2} .
$$

Les expressions (4) constituent, pour les inconnues $E_{n}$ et $F_{n}$, un système linéaire d'équations du type :

$$
\begin{aligned}
& \left(1+\frac{\omega_{1}^{2} T^{2}}{2} \frac{S_{n}}{D_{n}}\right) E_{n}+\frac{\omega_{1}^{2} T^{2}}{2} \frac{T_{n}}{D_{n}} F_{n}+\frac{\omega_{1}^{2} T^{2}}{2} \sum_{m \neq n} \frac{1}{D_{m}}\left(S_{m} E_{m}+T_{m} F_{m}\right)=M_{0} J_{-n}(x) \\
& \left(1+\frac{\omega_{1}^{2} T^{2}}{2} \frac{P_{n}}{D_{n}}\right) F_{n}+\frac{\omega_{1}^{2} T^{2}}{2} \frac{Q_{n}}{D_{n}} F_{n}+\frac{\omega_{1}^{2} T^{2}}{2} \sum_{m \neq n} \frac{1}{D_{m}}\left(P_{m} E_{m}+Q_{m} F_{m}\right)=0
\end{aligned}
$$

où $S_{n}, T_{n}, P_{n}, Q_{n}$ sont des expressions qui contiennent les fonctions de Bessel.

Pour calculer la courbe de résonance pour l'absorption de $\bar{n}$ photons $\Omega$, on peut conserver seulement les termes $E_{\bar{n}}$ et $F_{\bar{n}}$ qui contiennent le dénominateur $D_{\bar{n}}$ résonnant à $\omega_{0}=\omega+\bar{n} \Omega$, dans l'hypothèse où $\omega^{2} \mathrm{~T}^{2} /\left(1+\Omega^{2} T^{2}\right) \ll 1$. Cette condition est très bien satisfaite dans la situation de nos expériences. Le système (5) ainsi simplifié se réduit à deux équations à deux inconnues $E_{\bar{n}}$ et $F_{\bar{n}}$, par lesquelles on calcule les expressions de l'aimantation et du couple pour la résonance à $\overline{\mathrm{n}}$ photons. L'approximation que l'on utilise pour résoudre les équations de Bloch est meilleure que celle qui consiste à considérer seulement quelques termes dans l'expression de $M_{z}$. Dans notre cas on obtient pour chaque résonance une solution qui néglige seulement l'effet des autres résonances. L'absorption totale des spins est obtenue en sommant les différentes raies de résonance et la seule approximation introduite dans tout le procédé est dans le calcul de la solution pour chaque résonance. Au contraire en considérant quelques termes dans $M_{z}$ on obtient une solution qui considère en même temps les raies de résonance et l'effet de superposition, mais non exactement.

Les équations de Bloch modifiées ont été résolues avec la même technique. On déduit aussi dans ce cas les expressions des termes $E_{n}$ et $F_{n}$ desquels on tire le couple dû à la résonance à $\mathrm{n}$ photons :

On obtient :

$$
\begin{aligned}
C_{n}=\frac{\chi_{0} H_{1}^{2} J_{n}^{2}(x)}{\Delta_{n}} & \left\{\left(\omega+n \Omega+\delta_{n}\right) T+\omega_{0} T \frac{\omega_{1}^{2} T^{2}}{2} \sum_{K} \frac{J_{n+K}(x)}{1+K^{2} \Omega^{2} T^{2}}\left[J_{n+K}(x)-J_{n-K}(x)\right]\right\}- \\
& -\frac{\chi_{0} H_{1}^{2} J_{n}(x)}{\Delta_{n}} \frac{\Omega T}{1+\Omega^{2} T^{2}}\left\{n J _ { n } ( x ) \left[1+\frac{\omega_{1}^{2} T^{2}}{2} \sum_{K} \frac{J_{n+K}(x)}{1+K^{2} \Omega^{2} T^{2}} \times\right.\right. \\
& \left.\left.\times\left(J_{n+K}(x)-J_{n-K}(x)\right)\right]+\frac{\Omega_{1} T}{2}\left(J_{n+1}(x)-J_{n-1}(x)\right)\left(\Delta \omega+n \Omega+\delta_{n}\right) T\right\}
\end{aligned}
$$

où

$$
\Delta_{n}=1+\left(\omega+n \Omega+\delta_{n}-\omega_{0}\right)^{2} T^{2}+\omega_{1}^{2} T^{2} \sum_{K} \frac{J_{n+K}^{2}(x)}{1+K^{2} \Omega^{2} T^{2}}
$$

et

$$
\delta_{n}=-\frac{\gamma^{2} H_{1}^{2}}{2} \sum_{K} \frac{K \Omega T^{2}}{1+K^{2} \Omega^{2} T^{2}} J_{n-K}^{2}(x) .
$$

Dans ces expressions nous avons fait apparaître la fréquence $\Omega$ dans l'argument des fonctions de Bessel, puisque expérimentalement la fréquence est fixée et l'on balaye le champ statique $H_{0}$.
Dans l'expression du couple on observe que le dénominateur $\Delta_{n}$ pour la transition à $\mathrm{n}$ photons est résonnant lorsque

$$
\omega_{0}=\omega_{0 \mathrm{n}}=\omega+n \Omega+\delta_{n} .
$$


Aux valeurs très faibles de $\mathscr{H}_{1}$, on obtient :

$$
\omega_{0}=\bar{\omega}_{0 n}=\omega+n \Omega-\frac{\gamma^{2} H_{1}^{2}}{2} \frac{n \Omega T^{2}}{1+n^{2} \Omega^{2} T^{2}} .
$$

Alors que pour le balayage de $H_{\mathrm{e}}$ la séparation en fréquence entre les raies est $\Omega$, la présence du champ $H_{1}$ va donner pour le balayage de $H_{0}$ un petit décalage dans la position des résonances. En fonction du champ $\mathscr{H}_{1}$ il y a un déplacement radiatif dans la valeur résonnante :

$$
\begin{aligned}
& \Delta \omega_{0 \mathrm{n}}=\omega_{0 \mathrm{n}}-\bar{\omega}_{0 \mathrm{n}}= \\
& =-\frac{\gamma^{2} H_{1}^{2}}{2} \Omega T^{2}\left(\sum_{K} \frac{n-K}{1+(n-K)^{2} \Omega^{2} T^{2}} J_{K}^{2}(x)-\right. \\
& \left.\quad-\frac{n}{1+n^{2} \Omega^{2} T^{2}}\right) .
\end{aligned}
$$

De plus, comme le numérateur dans l'expression du couple est fonction de $H_{0}$, l'on obtient une asymétrie dans les courbes de résonance et un déplacement des maxima. On peut calculer que ce déplacement est à peu près $\Delta \omega_{0 \mathrm{n}} /[(\omega+n \Omega) T]$ et donc petit comparé à celui qui apparaît dans le dénominateur.

L'allure du déplacement radiatif en fonction de l'intensité du champ $\mathfrak{H}_{1}$ est très différente de celle que l'on obtient en appliquant simplement l'argument Bloch-Siegert pour les transitions à un ou plusieurs photons. En effet il faut rappeler que, dans notre expérience, on balaye le champ statique $H_{0}$ au lieu de balayer le champ effectif $H_{\mathrm{e}}$ et que la direction de relaxation de l'aimantation n'est pas parallèle au champ $H_{\mathrm{e}}$.

Pour comparer les résultats expérimentaux à la théorie, on a sommé pour chaque valeur de $H_{0}$ les contributions des différentes résonances à l'aide d'un ordinateur $\left(^{*}\right)$. On a alors déterminé la position et la valeur des maxima dans le couple total. La position des maxima est donnée par le déplacement radiatif de chaque résonance et par un effet de superposition entre les différentes raies. On a trouvé que les deux contributions sont tout à fait comparables. Pour isoler le déplacement radiatif on pourrait penser augmenter la fréquence $\Omega$, c'est-à-dire la séparation entre les raies. On obtiendrait alors une diminution de l'effet de superposition, mais la situation est presque la même, puisque le déplacement radiatif est inversement proportionnel à $\Omega$.

II. Etude expérimentale. - a) MONTAGe eXPÉRIMENTAL. - La technique expérimentale a été reportée ailleurs [9]. On en donnera ici une description sommaire. L'échantillon (1 gramme de DPPH comprimé de manière à former un cylindre), est suspendu à un fil de quartz de $10 \mu$ de diamètre, dans un tube en

(*) L'ordinateur IBM 7090 de l'Université de Pise. verre, où l'on a fait le vide. Le champ $H_{0}$ est vertical, parallèle au fil de torsion. Deux couples de bobines de Helmholtz produisent les champs radiofréquence de 64,3 et de $10 \mathrm{MHz}$ oscillant respectivement dans les directions perpendiculaire et parallèle au champ statique. Le champ statique est balayé par un potentiomètre tournant, et modulé à la fréquence propre $\mathrm{du}$ pendule de torsion ( 0,2 cycle par seconde). La rotation du pendule est détectée par une photocellule différentielle, qui reçoit la lumière réfléchie par un petit miroir solidaire au fil de torsion. Le signal à la sortie de la photocellule est détecté en phase et envoyé à l'axe $y$ d'un enregistreur, dont le déplacement $x$ est commandé par la tension de balayage du champ.

Le champ de radiofréquence perpendiculaire utilisé dans les expériences est un champ rectiligne tandis que dans la théorie seulement une composante tournante a été considérée. Néanmoins, comme $\omega_{1} \ll \omega$, le seul effet de la composante contre-tournante est un petit décalage Bloch-Siegert de l'ordre de $\gamma H_{1}^{2} / 4 H_{0}$, tout à fait négligeable. Pour obtenir l'intensité du champ à $64,3 \mathrm{MHz}$ on a mesuré la largeur de la résonance en l'absence du champ à $10 \mathrm{MHz}$. Puisqu'on a mesuré précédemment le temps de relaxation de cet échantillon de DPPH [10], la largeur de la raie permet de déduire l'intensité du champ de radiofréquence agissant sur l'échantillon. L'intensité de la composante tournante du champ de radiofréquence appliqué dans toute l'expérience vaut $0,5 \mathrm{G}$.

L'intensité relative du champ à $10 \mathrm{MHz}$ a été obtenue en observant la tension induite dans une bobine. On a déduit la valeur absolue en normalisant un point d'une courbe expérimentale sur la courbe théorique correspondante. L'intensité obtenue donne un excellent accord entre toutes les autres courbes correspondantes, expérimentales et théoriques.

b) RÉSULTATS EXPÉRIMENTAUX. - La figure 1 est un enregistrement du signal de sortie du détecteur de phase, en fonction de l'intensité du champ statique. Il représente avec une bonne approximation la dérivée par rapport à $H_{0}$ du couple mécanique $C$ agissant sur l'échantillon. On a marqué sur la figure la résonance centrale $C_{0}$, due à l'absorption d'un photon de fréquence $\omega$, et les raies satellites, dues à l'absorption d'un photon $\omega$ et à l'absorption ou à l'émission d'un, deux et trois photons de fréquence $\Omega$.

Sur la figure 2, on a reporté l'intensité relative des résonances en fonction de l'intensité du champ oscillant $\mathfrak{H}_{1}$. On peut observer l'allure caractéristique des fonctions de Bessel de différents ordres.

On a utilisé ces diagrammes d'intensité pour obtenir une mesure absolue du champ $\mathfrak{H}_{1}$. Précisément le point d'intensité zéro pour le couple $C_{0}$ (indiqué par une flèche sur la figure) a été choisi pour la normalisation des valeurs du champ oscillant.

Dans les figures 3 et 4 les déplacements des transitions à un et deux photons $\Omega$ sont portés en fonction de $\mathfrak{H}_{1}$. Ce que l'on a mesuré expérimentalement est la 

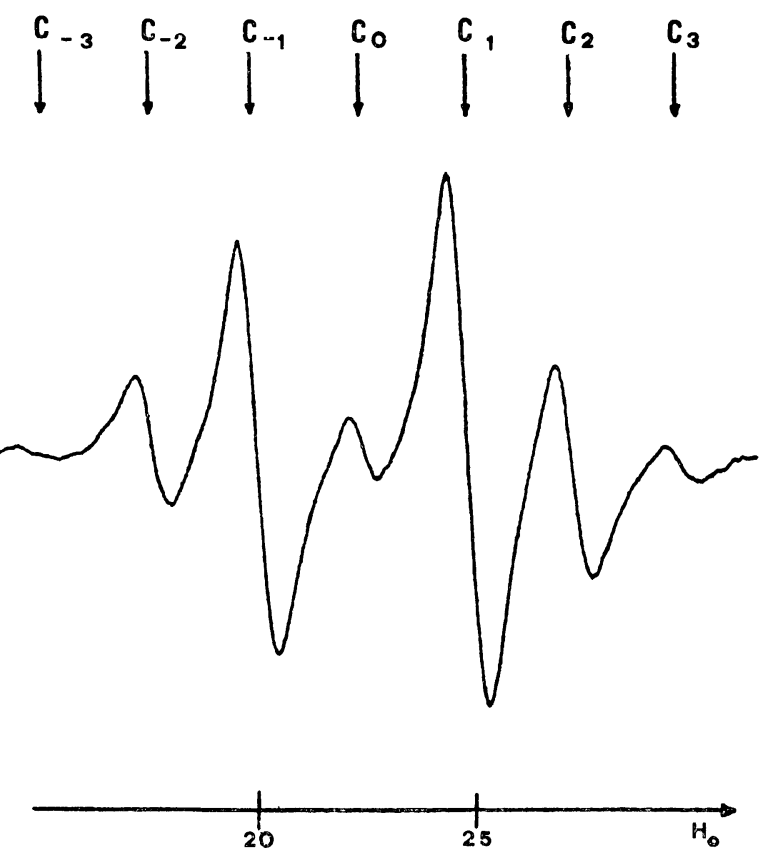

Fig. 1. - Enregistrement de la dérivée du couple agissant sur l'échantillon en fonction du champ magnétique $H_{0}$ en gauss avec un champ oscillant $\mathscr{H}_{1}$ de $7,4 \mathrm{G}$.
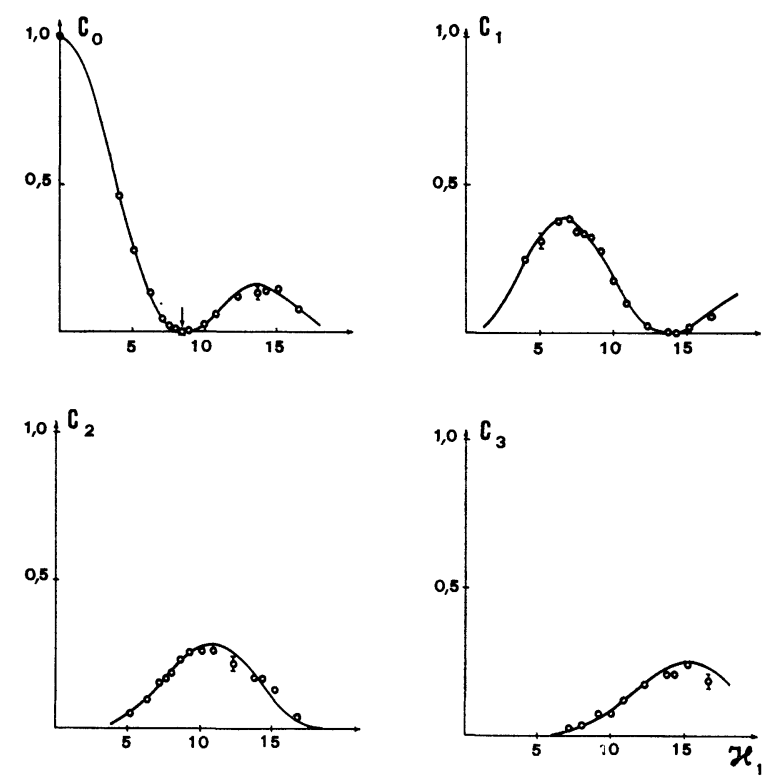

FIG. 2. - Intensité des résonances en fonction du champ $\mathfrak{H}_{1}$ en gauss, calculée relativement à la hauteur de la raie $C_{0}$ à champ $\mathfrak{J}_{1}$ nul. Le bruit mécanique du pendule et l'instabilité électronique sont compris dans l'erreur reportée.

distance entre les deux résonances à un photon $\left(C_{1}\right.$ et $\left.C_{-1}\right)$ (Fig. 3) et entre celles à deux photons $\left(C_{2}\right.$ et $\left.C_{-2}\right)$ (Fig. 4). Sur la figure 3 , pour certaines valeurs du champ $\mathfrak{H}_{1}$, il n'y a pas des résultats expérimentaux; pour ces valeurs l'intensité de raies à un photon est trop petite (Fig. 2) pour mesurer leur déplacement. On a reporté sur les figures 3 et 4 les courbes théoriques du déplacement radiatif des résonances (formule (7)) et celles obtenues par l'ordinateur qui

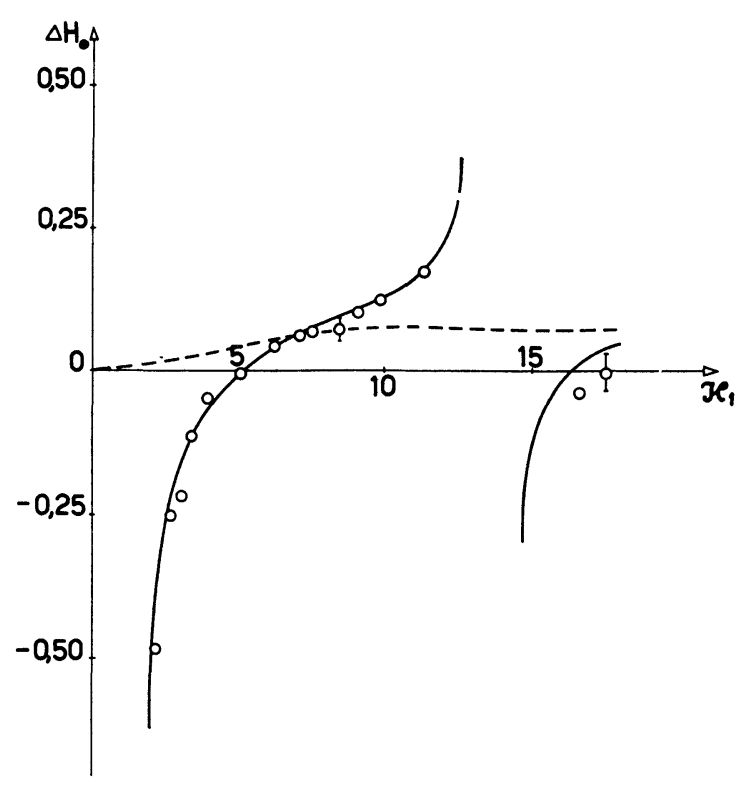

Fig. 3. - Points expérimentaux et courbe théorique du déplacement des transitions à un photon en fonction du champ $\mathfrak{H}_{1}$ en gauss. La courbe théorique pointillée représente le déplacement radiatif du maximum sans l'effet de superposition des autres raies. On a indiqué deux erreurs expérimentales différentes puisque pour les valeurs du champ $\mathfrak{H}_{1}$ supérieures à $15 \mathrm{G}$, il y a instabilité dans l'électronique sous l'influence de la puissance de radiofréquence.

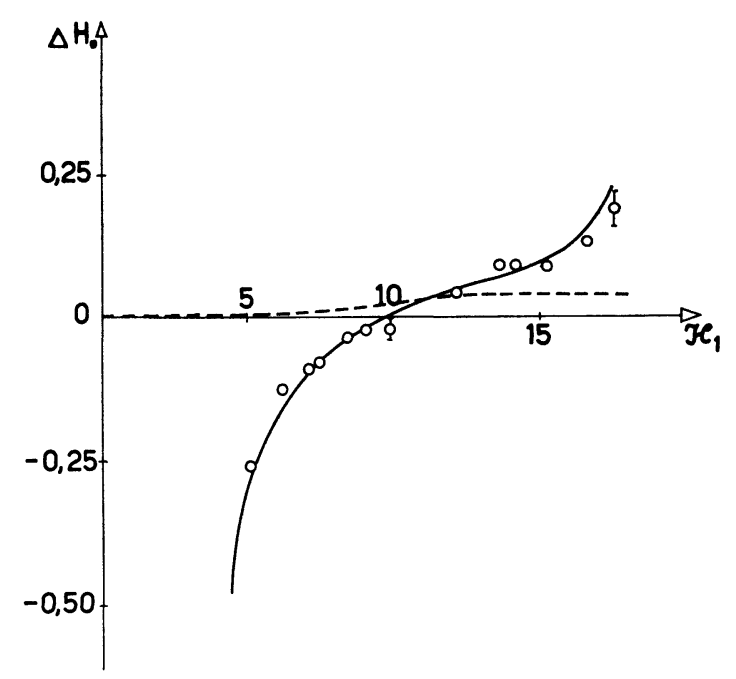

FIG. 4. - Points expérimentaux et courbes théoriques du déplacement des transitions à deux photons en fonction du champ $\mathfrak{H}_{1}$.

tiennent compte de l'effet de superposition entre les différentes raies. On observe que la contribution de la superposition est plus importante de celle du déplacement radiatif.

En considérant l'effet de la superposition, l'accord entre les courbes théoriques et les points expérimentaux est très bon, sauf pour deux points aux valeurs plus élevées de l'intensité du champ $\mathcal{J}_{1}$. Il y a peu de mesures dans cette région et l'erreur expérimentale est plus importante du fait que la résonance est plus 
faible et le bruit plus élevé, à cause de la forte puissance de radiofréquence. Néanmoins le désaccord entre les résultats expérimentaux et les valeurs théoriques ne peut être attribué aux erreurs expérimentales. On peut supposer que le temps de relaxation evientplus court. Puisque les conditions $\omega_{1} \tau_{\mathrm{c}} \ll 1$ et $\Omega_{1} \tau_{\mathrm{c}} \ll 1 \quad\left(\tau_{\mathrm{c}}\right.$ temps de corrélation égal à $5 \times 10^{-11}$ s) sont toujours bien satisfaites, le temps de relaxation ne doit pas dépendre de $\mathfrak{H}_{1}$.

Toutefois aux intensités plus élevées de $\mathfrak{H}_{1}$, l'échantillon s'échauffe et on peut penser à une diminution de $T$ avec la température. Supposant que le temps de relaxation est inversement proportionnel à la température et que la température de l'échantillon soit de $60^{\circ} \mathrm{C}$ on peut confirmer l'accord entre la théorie et l'expérience aussi pour ces valeurs du champ $\mathfrak{H}_{1}$.

Conclusion. - Les équations de Bloch donnent une description complète de la résonance magnétique dans le référentiel tournant pour le DPPH. Les résultats expérimentaux sont en parfait accord avec la théorie et l'on a confirmé la validité des expressions obtenues pour le déplacement des résonances, pour lequel la contribution de l'effet de superposition est plus grand que le déplacement radiatif.

Les auteurs tiennent à remercier le Professeur A. Gozzini pour des fructueuses discussions et pour sa lecture critique du manuscrit.

\section{Bibliographie}

[1] Benoit (H.), Pommier (J.), C. R. Acad. Sci., 1963, 256, 3647.

[2] Lhote (G.), Motchane (J. L.), Theobald (J. G.), C. R. Acad. Sci. Paris, 1964, 259, 1034.

[3] Abragam (A.), The Principles of Nuclear Magnetism, Oxford, Clarendon Press, 1961, 566.

[4] Gozzini (A.), Proc. XII Coll. Ampère 1963, North Holland Publ. Co., 1964, 82.

[5] Redfield (A. G.), Phys. Rev., 1955, 98, 1787.

[6] Anderson (W. A.), Phys. Rev., 1956, 102, 151 Novikov (L. N.), PoKazan'ev (V. G.) et YaKub (L. T.), Soviet Physics JETP, 1968, 26, 752.
[7] Schwerdtfeger (C. F.) et Koss (T. A.), Phys. Rev., 1969, 166, 259.

Koss (T. A.), Alexander (S.) et Schwerdtfeger (C. F.), Can J. Phys., 1968, 46, 1685.

[8] Codrington (R. S.), Olds (J. D.) et Torrey (H. C.), Phys. Rev., 1954, 95, 607.

Garstens (M. A.) et Kaplan (J. I.), Phys. Rev., 1955, 99, 459.

[9] Vladimirskil (K.), Soviet Physics JETP, 1958, 6, 412.

[10] Alzetta (G.), Arimondo (E.), Ascoli (C.) et GozzINI (A.), Nuovo Cimento, 1967, 52B, 392. 